BULLETIN OF THE

AMERICAN MATHEMATICAL SOCIETY

Volume 80, Number 4, July 1974

\title{
AUTOMORPHIC MAPPINGS IN $R^{n}$
}

\author{
BY O. MARTIO AND U. SREBRO
}

Communicated by F. W. Gehring, December 12, 1973

1. By an automorphic mapping in $R^{n}$ we mean a continuous, open, discrete, and sense-preserving mapping $f$ from a domain $D$ in $R^{n}$ into $\bar{R}^{n}=R^{n} \cup\{\infty\}$ which satisfies $f \circ g=f$ for all $g \in G$ for some discrete group $G$ of $n$-dimensional Möbius transformations, $n \geqq 2$. The results presented here indicate differences (see §5) as well as similarities (see §4) between automorphic functions in $C$ and automorphic mappings of bounded dilatation in $R^{n}, n>2$. By mappings of bounded dilatation we mean quasimeromorphic (qm) mappings (cf. [MRV 1-2]).

2. Let $G$ be a discrete Möbius group acting on the unit ball $B^{n}$. For $x_{0} \in B^{n}$ which is not fixed by any element of $G \backslash\{$ id $\}$ the set $P=\left\{x \in B^{n}\right.$ : $\left.d\left(x, x_{0}\right)<d\left(x, g\left(x_{0}\right)\right), \forall g \in G \backslash\{\mathrm{id}\}\right\}$ is a normal fundamental polyhedron; $d$ denotes the hyperbolic distance. If the hyperbolic measure $V\left(B^{n} / G\right)$ of $B^{n} / G$ is finite, then every normal fundamental polyhedron $P$ has a finite number of $(n-1)$-faces and a finite number of boundary vertices $\left\{p_{1}, \cdots\right.$, $\left.p_{k}\right\}=\bar{P} \cap \partial B^{n}[\mathbf{S}]$. The last set is void when $B^{n} / G$ is compact. $P$ is said to be simple if for every boundary vertex $p \in \bar{P} \cap \partial B^{n}$ all the $(n-1)$-faces of $P$ which meet at $p$ are pairwise $G$-equivalent. By a recent result of Leon Greenberg (unpublished) it can be shown [MS] that if $V\left(B^{n} / G\right)<\infty$, then every point $b \in \partial B^{n}$ which is fixed by a parabolic element $g \in G$ is a boundary vertex of some simple fundamental polyhedron. A Möbius transformation is called parabolic if it has a unique fixed point in $\bar{R}^{n}$.

Complete proofs of the following theorems and related results will appear in [MS].

3. The existence of automorphic meromorphic functions for Möbius groups in $C$ is usually proved by methods which cannot be used in $R^{n}$, $n>2$. However, with a suitable modification of a construction by J. W. Alexander [A] we obtain

THEOREM 1. Every discrete Möbius group acting on $B^{n}$ with $V\left(B^{n} / G\right)<\infty$ has qm automorphic mappings.

AMS (MOS) subject classifications (1970). Primary 30A60; Secondary 30A68, 57A99, 31B15. 
We do not know, whether qm automorphic mappings exist for all discrete Möbius groups in $\boldsymbol{B}^{n}$.

4. Let $G$ be a discrete Möbius group acting on $B^{n}$ with $V\left(B^{n} / G\right)<\infty$, $P$ a simple fundamental polyhedron, $\widetilde{P}$ a fundamental set for $G$ with $P \subset \tilde{P} \subset \bar{P}, f: B^{n} \rightarrow \bar{R}^{n}$ an automorphic qm mapping under $G$ and $N(f, \widetilde{P})=$ sup card $f^{-1}(y) \cap \tilde{P}$ over all $y \in \bar{R}^{n}$.

THeOREM 2. Let $f, G, P$, and $\widetilde{P}$ be as above.

(i) If $U$ is any open set in $R^{n}$ which meets $\partial B^{n}$, then $\bar{R}^{n} \backslash f\left(U \cap B^{n}\right)$ is of zero n-capacity.

(ii) If $N(f, \widetilde{P})<\infty$, then $\bar{R}^{n} \mid f B^{n}$ is of finite cardinality.

(iii) If $\bar{P} \subset B^{n}$ or if $\lim f(x)$, as $x \rightarrow p$ in $\tilde{P}$, exists at every boundary vertex $p \in \bar{P} \cap \partial B^{n}$, then $N(f, \widetilde{P})<\infty$ and

$$
\sum_{x \in f^{-1}(y) \cap \tilde{P}} i(x, f) / N(x, G)=N(f, \tilde{P})
$$

for all $y \in f B^{n}$. Here $i(x, f)$ denotes the local topological index of $f$ at $x$ and $N(x, G)=\operatorname{card}\{g \in G: g(x)=x\}$.

(iv) If $\bar{P} \cap \partial B^{n} \neq \varnothing$ and $N(f, \widetilde{P})<\infty$, then the set $Q$ of all parabolic fixed points of $G$ is dense in $\partial B^{n}$ and $f$ has a radial limit at every point $b \in Q$.

THEOREM 3. Let $f, G, P$, and $\widetilde{P}$ be as above and let $p \in \partial B^{n}$ be a boundary vertex of $P$. If $N(f, \widetilde{P})<\infty$ and $\lim f(t p)=a \neq \infty$ as $t \rightarrow 1$, then for all sufficiently small $r>0$

$$
A_{1} e^{-\alpha / r} \leqq M(r) \leqq A_{2} e^{-\beta / r} .
$$

Here $M(r)=\sup |f(x)-a|$ over all $x \in B^{n}$ with $|x-(1-r) p|=r, \alpha$ and $\beta$ are constants which depend on $n, G, N(f, \widetilde{P})$, and the dilatations of $f$, and $A_{1}, A_{2}$ are constants depending on $f$ and $G$.

The main tools used in the proofs of Theorems 2 and 3 are two capacity inequalities for condensers in $B^{n} / G$ and general results on open and discrete mappings on manifolds.

5. One of the differences between plane and space qm mappings $f$ is in the structure of their branch set $B_{f}$ (the set of points where $f$ is not a local homeomorphism) (cf. [Z], [MRV3, 2.3]). These results combined with information on the geometry of Möbius groups give

THEOREM 4. Let $f: B^{n} \rightarrow \bar{R}^{n}, n>2$, be a qm automorphic mapping for a Möbius group $G$ acting on $B^{n}$ with $V\left(B^{n} / G\right)<\infty$. If $\infty \notin f B^{n}$ or if $N(f, \widetilde{P})<$ $\infty$, then $B_{f} \neq \varnothing ;$ moreover $\partial B^{n} \subset \bar{B}_{f}$.

The condition $n>2$ is essential (the elliptic modular function is a counterexample), and so is the condition $V\left(B^{n} / G\right)<\infty$. This is shown by 
an example of a bounded qm local homeomorphism which is automorphic under an infinite Möbius group $G$ with $V\left(B^{n} / G\right)=\infty$.

\section{REFERENCES}

[A] J. W. Alexander, Note on Riemann spaces, Bull. Amer. Math. Soc. 26 (1920), 370-372.

[MRV1] O. Martio, S. Rickman and J. Väisälä, Definitions for quasiregular mappings, Ann. Acad. Sci. Fenn. Ser. A I No. 448 (1969), 40 pp. MR 41 \#3756.

[MRV2] —, Distortion and singularities of quasiregular mappings, Ann. Acad. Sci. Fenn. Ser. A I No. 465 (1970), 13 pp. MR 42 \#1995.

[MRV3] — , Topological and metric properties of quasiregular mappings, Ann. Acad. Sci. Fenn. Ser. A I No. 488 (1971), 31 pp.

[MS] O. Martio and U. Srebro, Automorphic quasimeromorphic mappings in $R^{n}$, Acta Math. (to appear).

[S] A. Selberg, Recent developments in the theory of discontinuous groups of motions of symmetric spaces, Proc. Fifteenth Scand. Congress (Oslo, 1968), Lecture Notes in Math. vol. 118, Springer-Verlag, Berlin, 1970, pp. 99-120. MR 41 \#8595.

[Z] V. A. Zorič, A theorem of M. A. Lavrent'ev on quasiconformal space maps, Mat. Sb. 74 (116) (1967), 417-433=Math. USSR Sb. 3 (1967), 389-404. MR 36 \#6617.

Department of Mathematics, University of Helsinki, Helsinki, Finland

Department of Mathematics, Technion, Haifa, Israel 